\title{
Including Credibility and Expertise in Group Decision- Making Process: An Approach Designed for UbiGDSS
}

\author{
João Carneiro $^{1,2}$, Diogo Martinho ${ }^{1}$, Goreti Marreiros ${ }^{1}$, and Paulo Novais ${ }^{2}$ \\ ${ }^{1}$ GECAD, Institute of Engineering - Polytechnic of Porto, Porto, Portugal \\ \{jomrc, diepm, mgt\} @i sep. ipp.pt \\ ${ }^{2}$ ALGORITMI Centre, at University of Minho, Braga, Portugal \\ pjon@di.uminho.pt
}

\begin{abstract}
Supporting group decision-making when the decision-makers are spread around the world is a complex process. The mechanisms of automated negotiation, such as argumentation, can be used in Ubiquitous Group Decision Support Systems to help decision-makers find a solution based on their preferences. However, there are some other important issues that affect the decisionmaking process beyond typical preferences over criteria and alternatives. In this paper, we propose an algorithm that will allow agents to reason about self-expertise and other decision makers' credibility. This way, we intend agents to achieve better quality and more consensual decisions. Our algorithm includes not only the decision-maker's preferences but also his intentions in the process. By using the proposed model, agents achieved a stronger consensus in all scenarios that were considered and higher satisfaction levels in the most complex scenarios.
\end{abstract}

Keywords: Group Decision Support Systems, Credibility, Expertise, Decision Satisfaction, Multi-Agent Systems

\section{Introduction}

The future and success of organizations depend greatly on the quality of every decision made. It is known that most of the decisions in organizations are made in groups [1]. To support this type of decision, Group Decision Support Systems (GDSS) have been widely studied throughout the last decades [2,3]. However, in the last decades, we have seen a remarkable change in the context where/how the decision-making process happens, especially in large organizations $[4,5]$. With the appearance of global markets, the growth of multinational enterprises and a global vision of the planet, we find chief executive officers and top managers (decision-makers) spread around the world, in different countries and with different time zones. To provide an answer and operate correctly in this type of scenarios traditional GDSS have evolved to what we identify today as Ubiquitous Group Decision Support Systems (UbiGDSS). UbiGDSS support the decision-making process by using main characteristics of ubiquity ("anytime" and "anywhere") [6].

Supporting the group decision-making process when decision-makers are dispersed is a complex task [7]. It is obvious that most of techniques that have been proposed in 
the literature do not take advantage of the recognized benefits inherent to group decision-making [8]. It is very important to read the literature under the topic of group decision-making $[8,9]$ when the computer science researchers intend to develop technologies to support groups, especially in ubiquitous scenarios. The benefits associated to group decision-making are very clear. For instance, [8] stated some advantages, such as: to share workloads, to build social networks, to gain support among stakeholders, to train less experienced group members and most importantly to improve the quality of the decision. However, even if these benefits seem to be rather obvious to most people, sometimes researchers forget the conditions necessary to attend them. Group interaction should be promoted to improve the quality of ideas and solutions $[10,11]$. The group decision-making process improves members ability to learn and also stimulates their cognition level $[12,13]$. Moreover, studies [9] show that cognitive stimulation helps people to think of new ideas, "unique combination of sub ideas, or a complex solution whose total value is greater than the sum of its parts".

It is known that a group's ability to recognize the expertise of its members can be vital to its success $[14,15]$. McGrath [16] stated that a major resource available to the problem-solving group is the expertise of the group members. Steiner [17] considered that decision-makers must coordinate and use their resources as efficiently as possible to take maximum advantage of the group capabilities. Using a large amount of resources may not be enough if the group fails to use them wisely. Bonner, Baumann and Dalal [18] stated that these resources include: individual competencies, skills, and knowledge of group members. Moreover, it is important for decision-makers to be able to identify status of hierarchies and leaderships [19]. In fact, Kameda, Tindale and Davis [20] stated that "distinguishing two levels of inputs, namely, preference and cognition, provides a useful overarching conceptual picture for synthesizing our empirical knowledge about decision making in consensus groups" and in the same work they concluded that "social sharedness plays a vital, perhaps the most critical, role in determining actual consensus processes and outcomes".

In this work, we study how the decision-making group can achieve stronger consensus and more satisfactory decisions by making agents able to reason about self-expertise and other decision-makers' credibility. For that, we propose an algorithm that uses the decision-maker problem's configuration, in terms of expertise level (of its own), credibility (of other decision-makers) and behaviour style (of its own). In the first place, the algorithm enables the agent to create a sub-group which includes all the decisionmakers considered to be credible plus himself. In the second place, the algorithm checks if there are any alternatives with more supporters than his preferred one(s). If so, the agent will weigh this difference with the values related to the dimensions of "Concern for Self" and "Concern for Others" which are part of his behaviour style. For this, the agent integrates his expertise level and the assessments performed to the alternatives in the equation. If the alternative being analysed becomes a better choice at a certain instant of the decision-making process, the agent includes this new alternative in his group of solutions that may be accepted. We have anticipated that agents able to identify new possible alternatives and redefine objectives were more flexible, and therefore could achieve better quality decisions and achieve stronger consensus. 
The rest of the paper is organized as follows: in the next section our approach is presented, where the algorithm and the necessary methods are described. In the section 3 we present the evaluation done to our work and report the results obtained. Finally, some conclusions are taken in section 4 , along with the work to be done hereafter.

\section{Methods}

Our method allows identifying situations where it makes sense for an agent to accept new alternatives as possible solutions for the problem. For that, the agent uses information provided by the decision-maker for the problem which includes expertise level, credibility and behaviour style. This information is very easy to configure and should follow the template proposed in [21]. This template proved to have a low configuration cost with an average configuration time of less than 5 minutes per individual.

The behaviour style corresponds to the intended behaviour of the decision-maker. In some of our previous works, we studied the difference between using the decisionmaker's personality and to let the decision-maker select the conflict style or the behaviour that he intends to us. In [22], we considered 5 behaviour styles differentiated by 4 dimensions.

Although there is not a universal definition found in literature for credibility it is still possible to relate it to the area of operation [23]. In the context of this work, it was considered the definition proposed by Flanagin and Metzger [23] when they say that "the overarching view is that credibility is the believability of a source or message, which is made up of two primary dimensions: trustworthiness and expertise". These dimensions differ from each other since trustworthiness refers to subjective components and expertise refers to objective components. The notion of credibility is related to other concepts including trust, reliability, reputation, etc. [23]. In our work, the main idea is to let a decision-maker choose which other decision-maker he considers to be credible towards a certain topic. This credibility evaluation is related with the concepts mentioned above and will be the reason why a decision-maker may consider another decision-maker to be credible in a certain topic and not in another (for example, with the related expertise level recognized for that decision-maker), and why a decision-maker may always consider another decision-maker as credible despite of the topic's difference (for example, due to reasons such as authority, reputation, etc.).

In our work, the decision-maker should make a self-evaluation about his expertise level for the topic at hand. We have considered the existence of five different expertise levels: Expert, High, Medium, Low and Null (the definition of the expertise levels definitions and values can be consulted in Table 1).

Our algorithm uses the problem configuration provided by the decision-maker, in terms of expertise level (of its own), credibility (of other decision-makers) and behaviour style (of its own). In the first place, the algorithm enables the agent to create a subgroup which includes all credible decision-makers plus himself.

In the second place, the algorithm checks if the agent's preferred alternative(s) is(are) less supported than other available alternatives. If so, the agent will weigh this 
difference with the values related to the dimensions of "Concern for Self" and "Concern for Others" which are part of his behaviour style.

Table 1. Expertise Levels

\begin{tabular}{lll}
\hline Expertise Level & Inverse of Expertise Level & Definition \\
\hline 5 & 1 & Expert \\
4 & 2 & High \\
3 & 3 & Medium \\
2 & 4 & Low \\
1 & 5 & Null \\
\hline
\end{tabular}

In the last place, the agent integrates his expertise level and the assessments performed to the alternatives in the equation. If the alternative being analysed demonstrates to be a better option during a certain instant of the decision-making process, the agent includes this new alternative in his group of solutions that may be accepted. Our algorithm can be consulted below (written in pseudocode):

- Let cAgsinfavourAlts be a list of alternatives supported by each credible agents $n$;

- Let selfPrefAlts be a list of alternatives already preferred/accepted by the agent where each alternative ap has associated a preference $p$;

- Let prefAlt be the current preferred alternative of the agent performing the evaluation where prefAlt $\in$ selfPrefAlts;

- Let max be an integer;

- Let resultPrefAlt, resultBestAlt, resultAlt be floats;

- Let bestalt be the alternative with higher result;

- Let altAlreadyPref be a boolean.

Begin

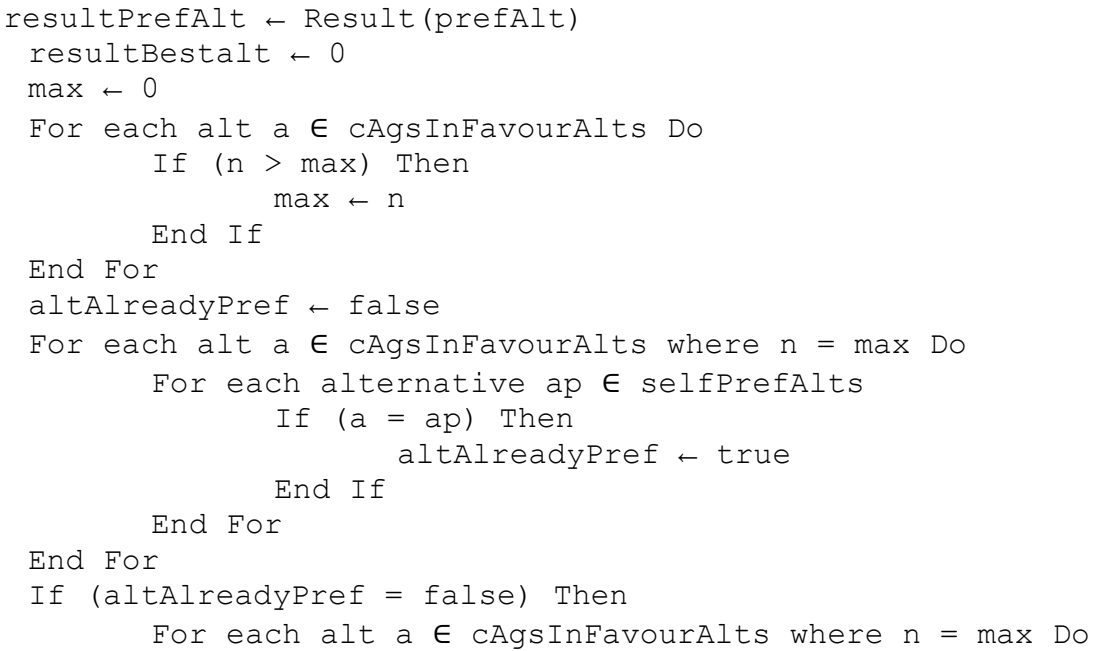




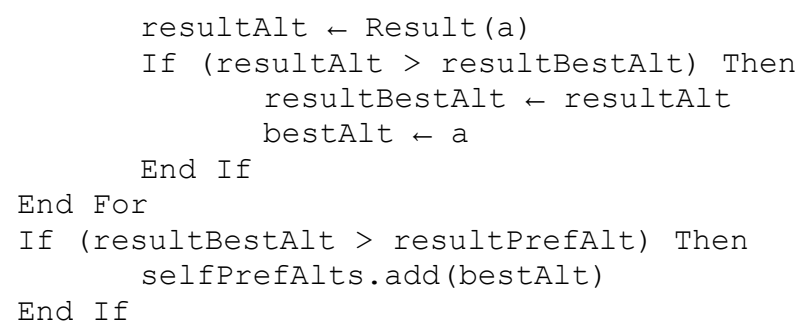

In order to measure the value of an alternative (in pseudocode it is equivalent to the Result() tag), we use formula (1). This formula intends to measure the value of an alternative at a certain instant for the agent. This measurement includes the agent's preferences and his intentions which correspond to the agent's behaviour style, expertise and credibility.

$$
A_{\text {Result }_{\text {Alt }}}=\frac{\operatorname{Alt}_{x} * C S * E+\left(\frac{N S}{N D}\right) * C O * I E}{C S+C O}
$$

$A l t_{x}$ is the assessment done to the alternative for which the result is being measured, $C S$ is the value of Concern for Self $(1,2,3]) E$ is the agent's Expertise Level, $N S$ is the current number of credible agents supporting $A l t_{x}, N D$ is the total number of credible participating agents, $C O$ is the value of Concern for Others $(1,2,3)$ and $I E$ is the agent's Inverse of the Expertise Level.

\section{Evaluation and Results}

The case that was studied involves agents' negotiation to solve the problem of choosing a desktop monitor for an organization that wants to purchase 200 new desktop monitors to one of its subsidiaries. Each agent represents one member of the organization administration board. Each alternative has been classified according to five criteria: Size (numerical, without value), Resolution (numerical, maximization), $\mathrm{Hz}$ (numerical, maximization), Ms (numerical, minimization) and Price (numerical, minimization).

In Table 2, all specifications are presented for each alternative considered. The satisfaction and consensus are used as metrics to evaluate the overall performance in different scenarios. The satisfaction metric is used to study the perceived quality (of the decision-maker that is represented) towards the chosen alternative or the alternative supported by most agents at a certain instant. For that, the notion of satisfaction that is used has been proposed in [24]. The satisfaction is measured in two parts (for agents without a defined behaviour only the first part is considered). It is first measured objectively through the formulas (2), (3) and (4). 
Table 2. Multi-Criteria Problem

\begin{tabular}{llllll}
\hline Alternatives & Size & Resolution & Hz & Ms & Price \\
\hline Asus 27" ROG SWIFT PG278Q & 27 & $2560 * 1440$ & 144 & 1 & $699,99 €$ \\
BenQ 27" XL2720Z & 27 & $1920 * 1080$ & 144 & 1 & $489,00 €$ \\
AOC 24" E2476VWM6 & 24 & $1920 * 1080$ & 60 & 1 & $154,90 €$ \\
BenQ 24" XL2430T & 24 & $1920 * 1080$ & 144 & 1 & $399,00 €$ \\
LG 27" 27MP37VQ-B & 27 & $1920 * 1080$ & 60 & 5 & $210,80 €$ \\
Asus LED 21.5" VS228HR & 21,5 & $1920 * 1080$ & 60 & 5 & $129,90 €$ \\
Samsung LED 22" S22C570H & 22 & $1920 * 1080$ & 60 & 5 & $179,90 €$ \\
BenQ 24" LED BL2420PT & 24 & $2560 * 1440$ & 60 & 5 & $399,90 €$ \\
Asus LED 24" VG248QE 144Hz 3D & 24 & $1920 * 1080$ & 144 & 1 & $288,90 €$ \\
Samsung 24" Curvo LED S24E500C & 24 & $1920 * 1080$ & 60 & 4 & $199,90 €$ \\
\hline
\end{tabular}

$$
\begin{gathered}
D_{\text {Lost }}=\text { Alt }_{F}-\text { Alt }_{P} \\
A_{\text {Conversion }}=2 A l t_{F}-1 \\
D_{\text {Satisfaction }}=\left(1-\left|A_{\text {Conversion }}\right|\right) * D_{\text {Lost }}+A_{\text {Conversion }}
\end{gathered}
$$

$D_{\text {Lost }}$ is the loss of the decision maker's satisfaction. This value corresponds to the difference between the assessments made for the alternative chosen by the group and for his preferred alternative. The loss is zero when the chosen alternative is the same as his preferred alternative. $A l t_{F}$ is the assessment made by the participant for the (final) alternative chosen by the group. $A l t_{P}$ is the assessment made by the participant for his preferred alternative and $A_{\text {Conversion }}$ is the conversion of the assessment made by the participant in the range $(-1 ; 1)$.

The second part relates the $D_{\text {Satisfaction }}$ and the behaviour defined by the decisionmaker. In this second part, the satisfaction is measured according to the values of the agent's defined behaviour for concern for self and concern for others dimensions. So, the $D_{\text {Satisfaction }}$ is remeasured using formula (5).

$$
D_{\text {Satisfaction }}=\frac{D_{\text {Satisfaction }} * C S+C A A D_{\text {Satisfaction }} * C O}{C S+C O}
$$

$C S$ is the value of Concern for $\operatorname{Self}(1,2,3), C A A D_{\text {Satisfaction }}$ is the average satisfaction of all the credible agents and $C O$ is the value of Concern for Others $(1,2,3)$.

The consensus level is measured with the value of the most supported alternative, at the time $t$, during iteration $i$, or round $r$.

To evaluate our model the average satisfaction and consensus levels were measured and compared in six simulation environments with three experiments (Table 3). Each experiment was performed in 200 simulations. The configurations were the same for each environment so that the results can be compared, however, these configurations have been randomly generated. In this first experiment, agents use the proposed algorithm but never change alternatives for each request $(\mathrm{CnE})$. In the second experiment, agents use the proposed algorithm and are able to perform changes over alternatives for 
each request (CnER). In the third experiment, agents use the argumentation model without the algorithm proposed in this work (WCnE).

The consensus level achieved in all environments in the experiments of "CnER" and "CnE" are very similar. On the other hand, consensus level obtained in the experiment of "WCnE" is a little bit lower. The average consensus values can be consulted in Table 3. The consensus level obtained by the experiment of "WCnE" decreases as the complexity of each simulation increases. In the last environment (40 Agents and 10 Alternatives) and the most complex, the difference between the average consensus values obtained in "WCnE" and "CnER" was of 0,184 . In the first environment (5 Agents and 5 Alternatives) and the least complex, the difference between the average consensus values obtained in "WCnE" and "CnER" was just of 0,036 .

Table 3. Average levels of consensus obtained

\begin{tabular}{llll}
\hline Simulation Environment & CnER & CnE & WCnE \\
\hline 5Ags and 5 Alts & 0,774 & 0,773 & 0,738 \\
5 Ags and 10 Alts & 0,683 & 0,67 & 0,633 \\
12 Ags and 5 Alts & 0,677 & 0,665 & 0,605 \\
12 Ags and 10 Alts & 0,662 & 0,64 & 0,559 \\
40 Ags and 5 Alts & 0,58 & 0,584 & 0,516 \\
40 Ags and 10 Alts & 0,574 & 0,56 & 0,39 \\
\hline
\end{tabular}

Fig. 1 shows that, the average consensus level obtained in "WCnE" is always inferior in each environment and the difference for each other experiment increases as the complexity also increases. It is also possible to verify that in environments using the same number of agents, the same difference increases if the number of alternatives that are considered also increases. Interestingly, however, even though the results obtained by experiments "CnER" and "CnE" towards the consensus level were very similar, Fig. 1 also shows that in environments where the number of agents is the same, agents using "CnER" will achieve a bit higher consensus (compared to "CnE") when more alternatives were considered (which increases the complexity of the environment).

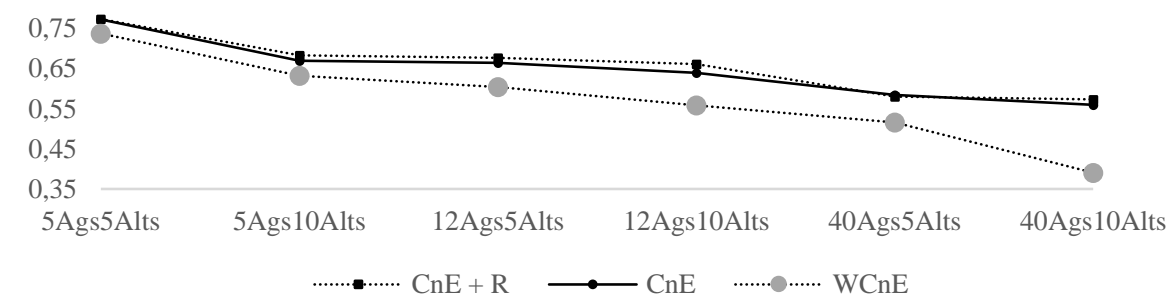

Fig. 1. Comparison between the average levels of consensus obtained in all experiments

Looking at the results related with the satisfaction level obtained, it is possible to verify that these values were once again very similar in experiments "CnER" and 
"CnE". This let us figure that agents that decide about which alternative should be requested, do not have advantage over agents without that ability. Table 4 presents the average satisfaction levels obtained in all experiments performed. In the first two environments agents "WCnE" obtained a bit higher average satisfaction level compared to agents "CnER" and "CnE". In the third and fourth environments, the average satisfaction levels were very similar and in the last two environments, agents "WCnE" obtained a bit lower average satisfaction level compared to agents "CnER" and "CnE"

Table 4. Average levels of satisfaction obtained

\begin{tabular}{llll}
\hline Simulation Environment & CnER & CnE & WCnE \\
\hline 5Ags and 5 Alts & 0,166 & 0,178 & 0,260 \\
5 Ags and 10 Alts & 0,199 & 0,202 & 0,266 \\
12 Ags and 5 Alts & 0,042 & 0,05 & 0,05 \\
12 Ags and 10 Alts & 0,067 & 0,062 & 0,094 \\
40 Ags and 5 Alts & $-0,044$ & $-0,042$ & $-0,075$ \\
40 Ags and 10 Alts & $-0,073$ & $-0,068$ & $-0,114$ \\
\hline
\end{tabular}

In Fig. 2 it is possible to verify the difference between the average satisfaction levels obtained in each experiment. However, we understood that as the complexity of the problem increases, agents "CnER" and "CnE" will achieve better results compared to "WCnE" (regarding the satisfaction level).

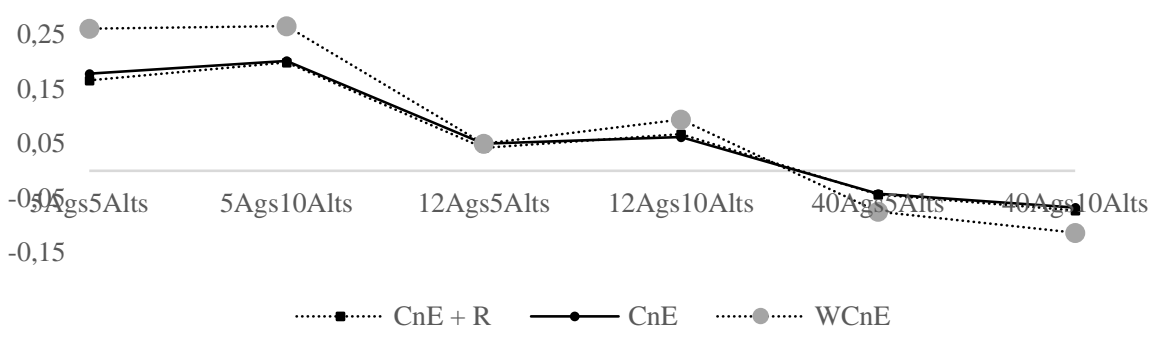

Fig. 2. Comparison between the average levels of satisfaction obtained in all experiments

\section{Conclusions and Future Work}

In this work, we propose an algorithm with the goal to make UbiGDSS that use negotiation models more intelligent. Our algorithm has the main goal to improve the quality of the decision made as well as the group capacity to achieve a stronger consensus. For that, agents that represent decision-makers can use information about typical problem's configuration, expertise level (of its own), credibility (of other decision-makers) and behaviour style (of its own). Using this information throughout the process let agents check if there are conditions to add new alternatives to their list of solutions that may be accepted. In addition, our algorithm uses a formula that identifies situations where 
agents should reformulate their objectives, and start to perform requests about a newly accepted alternative.

To test our algorithm a case of study was performed with six simulation environments that represent six different complexity levels. We concluded that agents that use the algorithm achieved higher average consensus levels when compared to agents that did not use it. We also concluded that as the context's complexity increases, the algorithm becomes even more important. In the most complex environment that we tested, agents that analysed credibility and expertise achieved a higher consensus $87,5 \%$ of the times while agents that did not use this model only achieved a higher consensus $8,5 \%$ of the times. When measuring the satisfaction level in the same environment agents with the ability to analyse credibility and expertise achieved a higher satisfaction level in $66 \%$ of the times. By combining both measures in the same study (satisfaction and consensus), it clearly shows the importance to allow agents to include the credibility and expertise in their analysis to obtain high quality decisions.

As future work, we intend to continue expanding our argumentation-based negotiation model. More precisely, we want to use at the same time both the algorithm presented in this paper and the model of the tendencies analysis presented in a previous study. We will publish a new version of our argumentation-based negotiation model with the main goal to promote interaction between decision-makers through a kind of dialogue that is perceptible to both humans and agents. This way we think that it is possible (together with automatic negotiation mechanisms) to achieve not only more consensual solutions but also with more quality. It is important that the system properly informs the decision-maker about each step of the negotiation process and the reasons behind suggestions that are given to him.

\section{Acknowledgements}

This work was supported by COMPETE Programme (operational programme for competitiveness) within Project POCI-01-0145-FEDER-007043, by National Funds through the FCT - Fundação para a Ciência e a Tecnologia (Portuguese Foundation for Science and Technology) within the Projects UID/CEC/00319/2013, UID/EEA/00760/2013, and the João Carneiro PhD Grant with the Reference SFRH/BD/89697/2012.

\section{References}

1. Luthans, F.: Organizational behavior. McGraw-Hill/Irwin 46, 594 (2011)

2. DeSanctis, G., Gallupe, B.: Group decision support systems: a new frontier. SIGMIS Database 16, 3-10 (1985)

3. DeSanctis, G., Gallupe, B.: A Foundation for the Study of Group Decision Support Systems. Management Science 33, 589-609 (1987)

4. Grudin, J.: Group Dynamics and Ubiquitous Computing. Communications of the ACM 45 , 74-78 (2002) 
5. Marreiros, G., Santos, R., Ramos, C., Neves, J.: Context-Aware Emotion-Based Model for Group Decision Making. Intelligent Systems, IEEE 25, 31-39 (2010)

6. Kwon, O., Yoo, K., Suh, E.: UbiDSS: a proactive intelligent decision support system as an expert system deploying ubiquitous computing technologies. Expert Systems with Applications 28, 149-161 (2005)

7. Cramton, C.D.: The mutual knowledge problem and its consequences for dispersed collaboration. Organization science 12, 346-371 (2001)

8. Dennis, A.R.: Information Exchange and Use in Group Decision Making: You Can Lead a Group To Information, But You Can'T Make It Think. MIS Quarterly 20, 433-457 (1996)

9. Hill, G.W.: Group versus individual performance: Are N+1 heads better than one? Psychological bulletin 91, 517 (1982)

10. Watson, W.E., Michaelsen, L.K., Sharp, W.: Member competence, group interaction, and group decision making: a longitudinal study. Journal of Applied Psychology 76, 803 (1991)

11. Hackman, J.R., Morris, C.G.: Group tasks, group interaction process, and group performance effectiveness: A review and proposed integration. Citeseer (1974)

12. Lamm, H., Trommsdorff, G.: Group versus individual performance on tasks requiring ideational proficiency (brainstorming): A review. European journal of social psychology 3, 361-388 (1973)

13. Osborn, A.F.: Applied Imagination; Principles and Procedures of Creative Problem-solving: Principles and Procedures of Creative Problem-solving. Scribner (1963)

14. Bottger, P.C., Yetton, P.W.: An integration of process and decision scheme explanations of group problem solving performance. Organizational behavior and human decision processes 42, 234-249 (1988)

15. Einhorn, H.J., Hogarth, R.M., Klempner, E.: Quality of group judgment. Psychological Bulletin 84, 158 (1977)

16. McGrath, J.E.: Groups: Interaction and performance. Prentice-Hall Englewood Cliffs, NJ (1984)

17. Steiner, I.D.: Group Process and Productivity (Social Psychological Monograph). (2007)

18. Bonner, B.L., Baumann, M.R., Dalal, R.S.: The effects of member expertise on group decision-making and performance. Organizational Behavior and Human Decision Processes $88,719-736(2002)$

19. Bonaccio, S., Dalal, R.S.: Advice taking and decision-making: An integrative literature review, and implications for the organizational sciences. Organizational Behavior and Human Decision Processes 101, 127-151 (2006)

20. Kameda, T., Tindale, R.S., Davis, J.H.: 14 Cognitions, Preferences, and Social Sharedness: Past, Present, and Future Directions in Group Decision Making. Emerging perspectives on judgment and decision research 458 (2003)

21. Carneiro, J., Martinho, D., Marreiros, G., Novais, P.: A General Template to Configure Multi-Criteria Problems in Ubiquitous GDSS. International Journal of Software Engineering and Its Applications 9, 193-206 (2015)

22. Martinho, D., Carneiro, J., Marreiros, G., Novais, P.: Dealing with Agents' Behaviour in the Decision-Making Process. In: Workshop Proceedings of the 11th International Conference on Intelligent Environments, pp. 4. IOS Press, (2015)

23. Flanagin, A.J., Metzger, M.J.: Digital media and youth: Unparalleled opportunity and unprecedented responsibility. Digital media, youth, and credibility 5-27 (2008)

24. Carneiro, J., Marreiros, G., Novais, P.: Using Satisfaction Analysis to Predict Decision Quality. International Journal of Artificial Intelligence ${ }^{\mathrm{TM}} 13,45-57$ (2015) 